\title{
The influence of acting mode of current on structure and mechanical properties of Ni-nanoTiN composite coatings
}

\author{
Menghua $\mathrm{Wu}^{1, \mathrm{a}}$, Jie $\mathrm{He}^{2, \mathrm{~b}}$ and Pengxiang $\mathrm{Lv}^{3, \mathrm{c}}$ \\ 1Dalian University, School of Mechanical Engineering, Dalian, 116622, China \\ ${ }^{2}$ Dalian University, School of Mechanical Engineering, Dalian, 116622, China \\ ${ }^{3}$ Dalian University, School of Mechanical Engineering, Dalian, 116622, China \\ a'wmh005@163.com, b1135513602@qq.com, 'Ipx_cn@163.com
}

Keywords: Ni-nanoTiN composite coatings; acting mode of current; microstructure; surface morphology; mechanical properties

Abstract. Ni-nanoTiN composite coatings were electrode posited on 45 steel substrate by using the ultrasonic-electrode position for nickel sulfamate bath. The effects of direct current, positive pulse current, reverse (positive and negative) pulse on surface morphology, binding force, micro hardness, friction and wear performance of Ni-nanoTiN composite coating. The results show that the micro hardness is $322.68 \mathrm{HV}_{0.1}$, the critical load of binding force is $25 \mathrm{~N}$, bigger cracks, rough surface, coarse grains and bad wearing of coating when direct current deposition; the micro hardness is 371.74 $\mathrm{HV}_{0.1}$, the critical load of binding force is $40 \mathrm{~N}$, smaller cracks, smooth surface, relatively small grain, better friction and wear performance of coating when positive current deposition; the micro hardness is $494.18 \mathrm{HV}_{0.1}$, the critical load of binding force is over $50 \mathrm{~N}$, non cracks, more smooth surface, tiny grains, best friction and wear performance of coating when positive and negative pulse deposition. Positive and negative pulse deposition may further refine grain, improve surface morphology, increase nanoTiN particles co-deposition and enhance mechanical properties of composite coating.

\section{Introduction}

With the development of electrode position technology, research on preparing composite coating has become focus and some research results have been used in practical production. Nowadays, the main improving methods of preparation composite coating are as follow: (1) changing the pulse current parameters (such as duty ratio, peak current density, etc.), (2) using organic additives (such as saccharin, coumarin, sodium dodecyl sulfate, etc.), (3) co-depositing TiN, SiC and other ceramics nano particles. The nucleation and growth of matrix metal grain during process of crystal may be controlled by applying pulse electrodepositing method, smaller grain size and more dense structure of depositing coating may also be gained[1-3]. Moreover, the physical and chemical properties of coatings can be improved, the excellent performance of the coating can be obtained, and the precious metals can be saved by changing the parameters of pulse current[4-6]. TiN is a metallic bonding compound with high melting point, low brittleness, high interface bonding strength and better chemical stability, etc, and the electrical conductivity of TiN is the highest in almost all nano ceramic particles[7]. $\mathrm{SiC}$ is a covalent bond compound, its matrix interface bonding strength and chemical stability are relatively poor compared with TiN. So TiN is normally co-deposited during electrode positing composite coatings for better reinforcing and wear-resisting effects[8]. Combining the characteristics of pulse electrode position and applying ultrasonic wave during electrode positing process, the effects of direct current, positive pulse, reverse (positive and negative) pulse on microstructure and properties of Ni-nano TiN composite coating were studied under the condition of unchanged bath composition and ultrasonic parameters. High resolution scanning electron microscope (SEM) was used to observe morphology of composite coating. The micro hardness, bonding force, friction and wear properties of composite coating prepared obtained with above three actions of current were analyzed and compared. 


\section{Experiment}

The depositing device. The depositing device is shown in Fig. 1. The temperature of bath was controlled between $45^{\circ} \mathrm{C}$ to $50^{\circ} \mathrm{C}$ by a constant-temperature water bath heater and $\mathrm{pH}$ of bath was between 4 to 5 , the power supply was an intelligent double pulse electrode plating power (GKDMZ12), the NC ultrasonic generator (KQ-1000DB, 300W) and electric adjustable speed mixer (D40-2F, 350r/min) were used to disperse and stir the bath during electrode positing process. Acting modes of current were direct current, positive pulse, reverse pulses and electrode positing time was 30 $\min$.

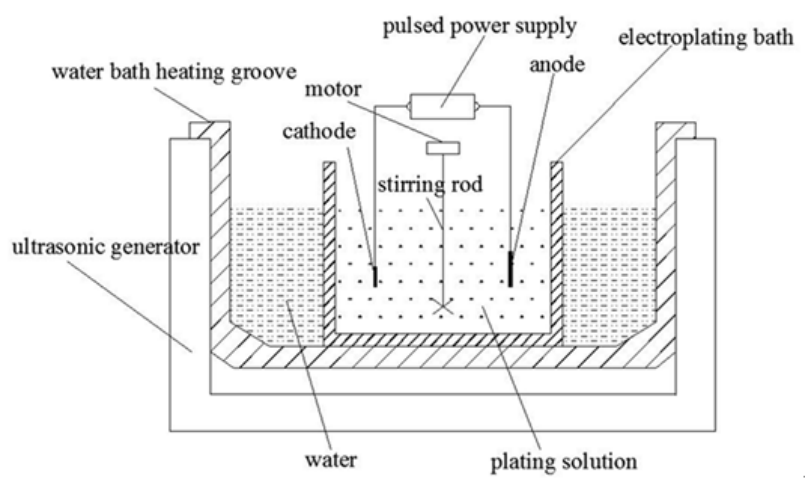

Fig. 1. Schematic of electrode positing device.

Experimental materials and test methods. 45 steel (size: $40 \mathrm{~mm} \times 30 \mathrm{~mm} \times 3 \mathrm{~mm}$ ) was used as the cathode and its effective depositing area was $0.09 \mathrm{dm}^{2}$, non-depositing area was sealed with insulating materials. Nickel plate (size: $60 \mathrm{~mm} \times 40 \mathrm{~mm} \times 3 \mathrm{~mm}$, purity: more than $99.99 \%$ ) was chosen as anode. The anode paralleled to the cathode and the inter distance between electrodes was $60 \mathrm{~mm}$. Nano TiN particles (average particle size: $60 \mathrm{~nm}$ ) was chosen as the second phase particles. The $\mathrm{pH}$ meter (PHSJ-4F) was used to test the $\mathrm{pH}$ of bath, the micro hardness meter (HXD-1000) was used to test the surface micro hardness of coatings under certain test conditions (load was $100 \mathrm{~g}$, loading time was $4 \mathrm{~s}$, the average value of five measure points was taken as the results), the scanning electron microscope (SEM, JES-5500LV) was used to observe surface morphology of composite coatings, the high load scratch instrument (Swiss, CSM) was used to measure bonding force of coatings under certain test conditions (single scrapes, initial load was $10 \mathrm{~N}$, ultimate load was $50 \mathrm{~N}$, loading method was linear, scratches rate was $5 \mathrm{~mm} / \mathrm{min}$, scratches length was $5 \mathrm{~mm}$, indenter tip radius was $200 \mu \mathrm{m}$ ), the friction-wear testing machine (MMW-1A) was used to test wear resistance of coatings under certain test conditions (dry friction, friction ring materials was 45 steel, loading force was $30 \mathrm{~N}$, rotating speed was $150 \mathrm{rpm}$, loading time was 180s).

Composition of bath and electrode positing parameters. The compositions of bath are shown in table 1. The preparation process of composite coating was as follows: washing $>$ degreasing with organic solvent $>$ ultrasonic cleaning $>$ clearing rust $>$ mechanical buffing $>$ mechanical Polishing $>$ washing $>$ activating $>$ washing $>$ ultrasonic-pulse electrode positing $>$ washing $>$ cleaning with anhydrous ethanol $>$ drying.

Table 1 Composition of bath

\begin{tabular}{lc}
\hline composition & $\begin{array}{c}\text { concentration } \\
(\mathrm{g} / \mathrm{L})\end{array}$ \\
\hline $\mathrm{Ni}\left(\mathrm{SO}_{3} \mathrm{NH}_{2}\right)_{2} \cdot 4 \mathrm{H}_{2} \mathrm{O}$ & 300 \\
$\mathrm{NiCl}_{2} \cdot 6 \mathrm{H}_{2} \mathrm{O}$ & 30 \\
$\mathrm{H}_{3} \mathrm{BO}_{3}$ & 30 \\
Sodiumdodecyl sulfate & 0.2 \\
$\mathrm{TiN}$ & 8 \\
\hline
\end{tabular}


Current wave form and parameters of pulse current. Current wave forms applied are shown in figure 2. The main parameters of pulse current were as follow: on time $\left(\mathrm{T}_{\mathrm{on}}\right)$, off time $\left(\mathrm{T}_{\text {off }}\right)$, positive working time (TF), negative working time (TR), Pulse period $T=T_{\text {on }}+T_{\text {off }}$, the positive and negative pulse reverse current of a cycle $T=T F+T R$, duty cycle $\gamma=T_{\text {on }} /\left(T_{\text {on }}+T_{\text {off }}\right)$, pulse frequency $f=1 / T$. The selected parameter values were as follow: $\mathrm{TF}=90 \mathrm{~ms}, \mathrm{TR}=10 \mathrm{~ms}$, current density $=6 \mathrm{~A} / \mathrm{dm}^{2}, \mathrm{~T}=1000 \mu \mathrm{s}$, $\mathrm{T}_{\text {on }}=250 \mu \mathrm{s}, \mathrm{T}_{\text {off }}=750 \mu \mathrm{s}$, calculated peak current $\mathrm{I}_{\mathrm{M}}=2.16 \mathrm{~A}$.

a

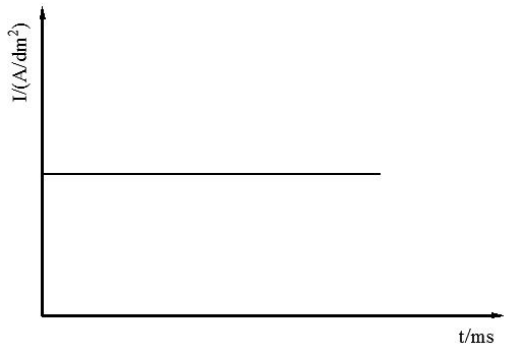

b

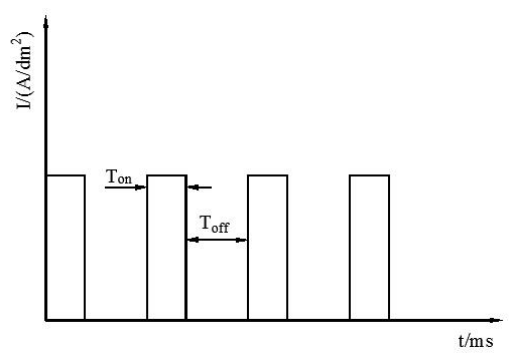

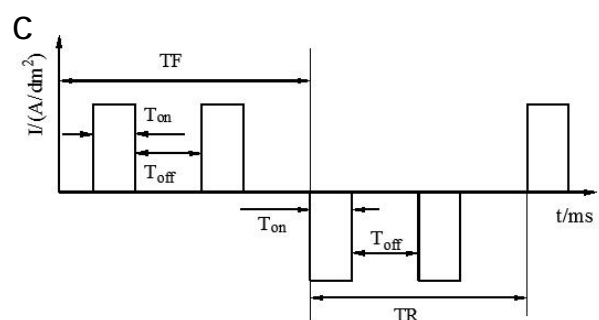

Fig. 2. Schematic of current waveform. (a) direct current; (b) positive pulse current; (c) reverse pulse current.

\section{The experimental results and discussion}

The influence of acting mold of current on morphology of coatings. Respectively applying direct current, positive pulse current, reverse (positive and negative) pulse current for electrode positing Ni-nanoTiN composite coatings under the condition of unchanged bath composition and ultrasonic parameters, the surface morphology (SEM) of coatings are shown in Fig. 3 and the cross section morphology (SEM) of coatings are shown in Fig. 4.

a
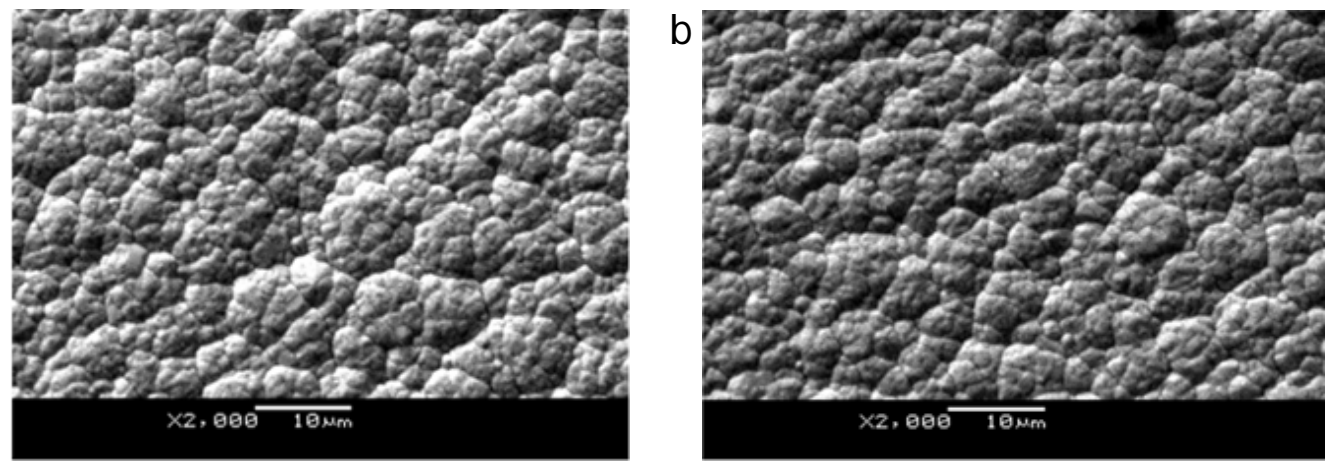

C

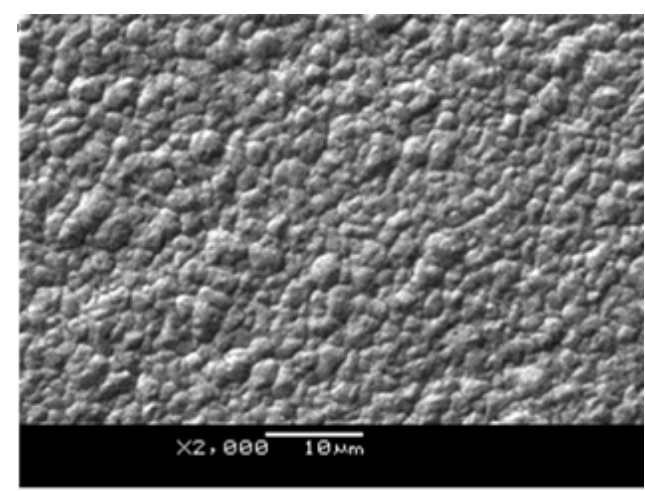

Fig. 3. The surface morphology (SEM) of electrode positing coatings under different acting mold of current. (a)direct current; (b) positive pulse current; (c) reverse pulse current.

By applying Image Tool software to calculate the grain size of coatings shown in Fig. 3, the result shown that the minimum average grain size of coatings was about $1 \mu \mathrm{m}$. It can be seen from the Fig. 3 : 
coarse grains and rough surface morphology of coating when applying direct current, smaller grain size and flat surface morphology of coating when applying positive pulse current, minimum grain size and more flat surface morphology of coating when applying reverse (positive and negative) pulse current.

The reasons for the above results are as follows: as the probability of crystal nucleation has close relationship with cathodic polarization, it is possible to form a new crystal nucleus when cathodic polarization is bigger; the formation of new crystal nucleus along with the growth of crystal nucleus, if the formation speed of new crystal nucleus is fast and the growth speed of crystal nucleus is slow, it is possible to obtain fine crystal coating[9]; as the peak current density is much higher than direct current under the condition of the pulse electrodepositing, so it may leads to high over-potential, result in nucleation rate of grain increasing significantly and formation speed of nucleation is greater than its growth speed, as a result, the grain of deposited layer is refined, dense and smooth coating is finally obtained.

It can be seen from Fig. 4 that the thickness uniformity of coating obtained under direct current electrode positing is poor and the inclusion phenomenon exists in the combination of coating and substrate, the thickness of coating obtained under positive pulse and reverse pulse electrode positing are uniform, the bonding surface of the coating and the substrate are smooth and flat.
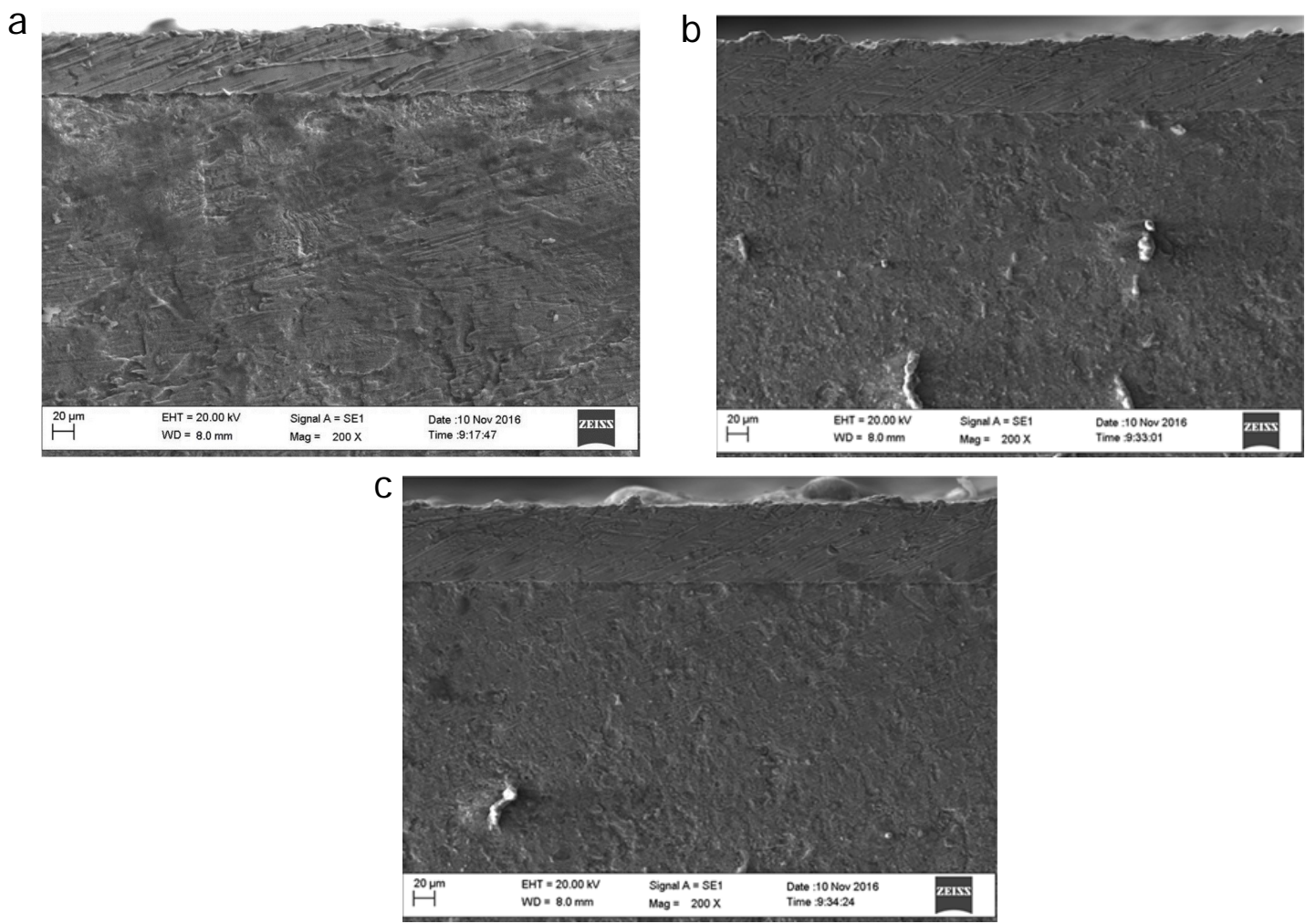

Fig.4. The cross section morphology (SEM) of electrode positing coatings under different acting mold of current. (a) direct current; (b) positive pulse current; (c) reverse pulse current.

The reasons for above results are as follows: due to the concentration polarization phenomenon of direct current depositing process, so the thickness of the coating is not uniform and exist inclusion phenomenon[10]; during positive pulse electrodepositing process, the growing grains will stop growth when the current is changed from positive to zero, the grain growth center may be shielded while pulse off time and force a new crystal nucleus form in each pulse action, the current is changed again from zero to positive followed by cycle, so the coating thickness may be uniform by effectively controlling the consistent growth rate of coating along the thickness direction; during reverse pulse electrodepositing process, in addition to the characteristics of a positive pulse electrode positing process, the raised coating and impurities produced during electrode position can be removed by 
action of negative pulse current, thus the coating thickness is more uniform and the binding surface is more smooth.

The influence of acting mold of current on co-depositing nano TiN particles. Respectively applying direct current, positive pulse current, reverse(positive and negative) pulse current for electrode positing Ni-nanoTiN composite coatings under the condition of unchanged bath composition and ultrasonic parameters, the distribution of TiN nano particles of coatings are shown in Fig. 5. It can be seen from the Fig. 5 that the content of TiN particle is small, the distribution of TiN particle is uneven and a certain degree of reunion of TiN particle in the coating obtained under direct current electrode positing; the content of TiN particle is increased, the uneven distribution of TiN particles is improved and reunion phenomenon is reduced to a certain extent in the coating obtained under positive pulse electrode positing; the content of nano TiN particle is relatively large, the distribution of TiN particles is evenly and reunion phenomenon is drastically reduced in the coating obtained under reverse pulse electrode positing.
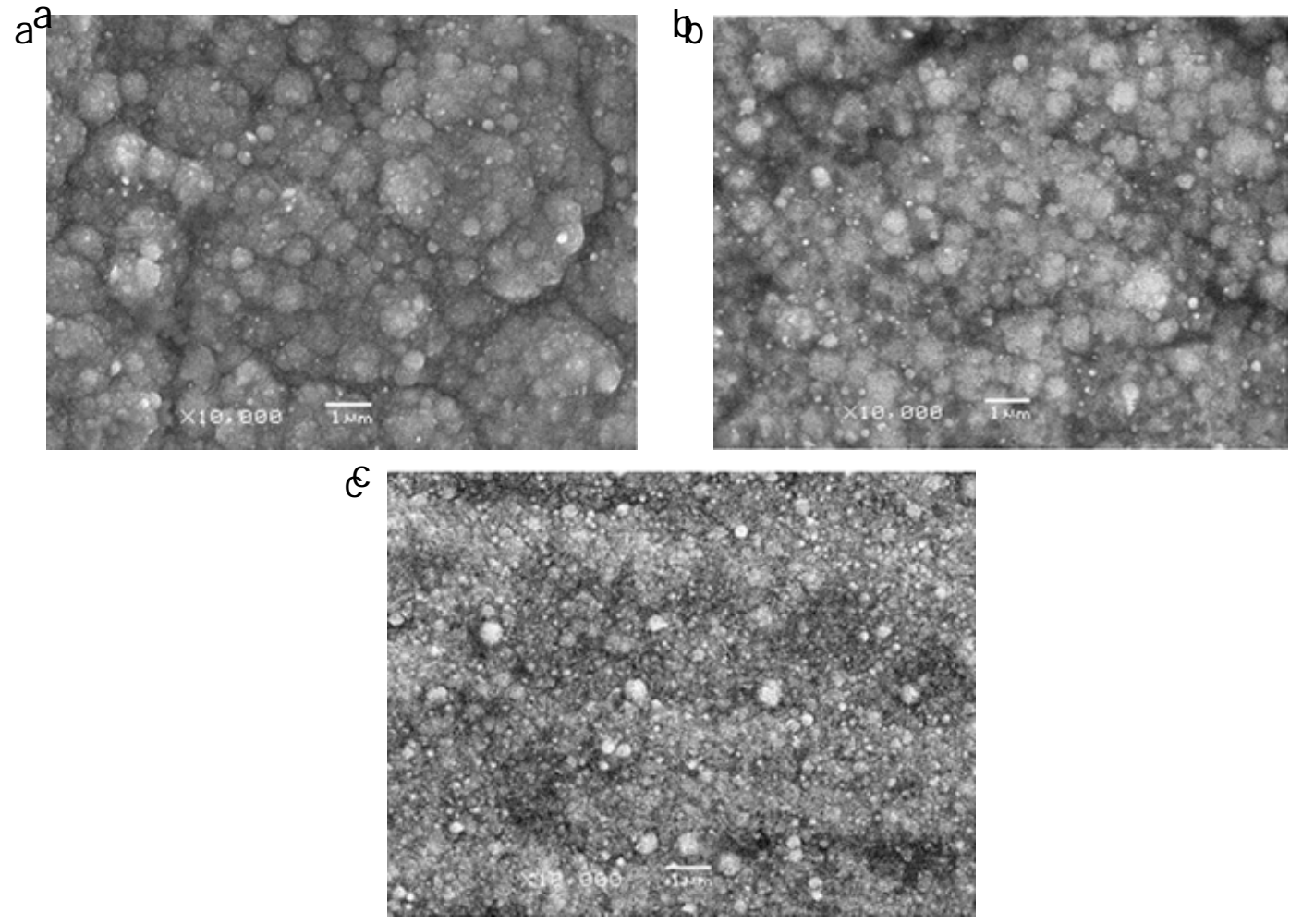

Fig.5. The distribution (SEM) of nano TiN particles in coatings under different acting mold of current. (a)direct current; (b) positive pulse current; (c) reverse pulse current.

The reasons for above results are as follows: during traditional direct current depositing process, due to concentration-polarization phenomenon, the ions around cathode cannot be timely replenishment and produce diffusion layer, so the co-deposition of nano TiN particles was affected; during positive pulse electrodepositing process, due to the concentration of ion on surface of cathode increased, the production of diffusion layer was inhibited to some extent, so more nano TiN particles co-deposited in coating; during reverse pulse electrodepositing process, the negative current made the micro convex of coating dissolved and the concentration of ion on surface of cathode was further improved, the production of diffusion layer was further inhibited and the thickness of diffusion layer was thinned, the cathode current efficiency was improved and the co-depositing rate of nano TiN particles was accelerated, so the content of nano TiN particles in coating increased and the grain of coating was more uniform and dense[11-12].

The influence of acting mold of current on binding force of coatings. The scratches photographs (observed at 50 times microscope) of the binding force of coating obtained under 
different acting mold of current are shown in Fig. 6. It can be seen from the Fig. 6 that larger fracture cracks appeared in scratch of coating obtained under direct current electrode positing when the critical load is $25 \mathrm{~N}$, tiny fracture cracks appeared in scratch of coating obtained under positive pulse electrode positing when the critical load is $40 \mathrm{~N}$, scratches was uniform and no fracture crack appeared in scratch of coating obtained under reverse current electrode positing when the critical load is $50 \mathrm{~N}$.

The reasons for above results are as follows: during traditional direct current depositing process, due to current had been always in a conducting state, it might lead to concentration of nickel ions near cathode could not be returned to normal levels, and it was easy to generate reaction of hydrogen evolution, then hydrogen evolution would heighten $\mathrm{pH}$ value of bath near cathodic area and formed basic salt or hydroxide, these substances may adsorbed on cathode or deposited in coating, it finally led to the decrease of the binding force between the coating and matrix; during positive pulse electrodepositing process, there was a certain concentration difference between nickel ions near cathode and in bath after the end of pulse conduction time, due to the actions of molecular dynamics and mechanical stirring, concentration of nickel ions near cathode would have a certain degree of recovery in the pulse off time, so it would inhibit the reaction of hydrogen evolution and finally improve the binding force of coating; during reverse pulse electrodepositing process, the negative current may dissolve a small amount of micro convex coating, and increase the concentration of nickel ion and the content of nano TiN particles near cathode, at same time, the negative current also may activate the cathode surface and enhance binding force of coating, so as to reduce content of impurity in coating and enhance performance of coating[13-14].So the binding force of composite coating obtained under reverse pulse electrodepositing is higher than the binding forces of composite coating obtained under direct current and positive pulse electrode positing.
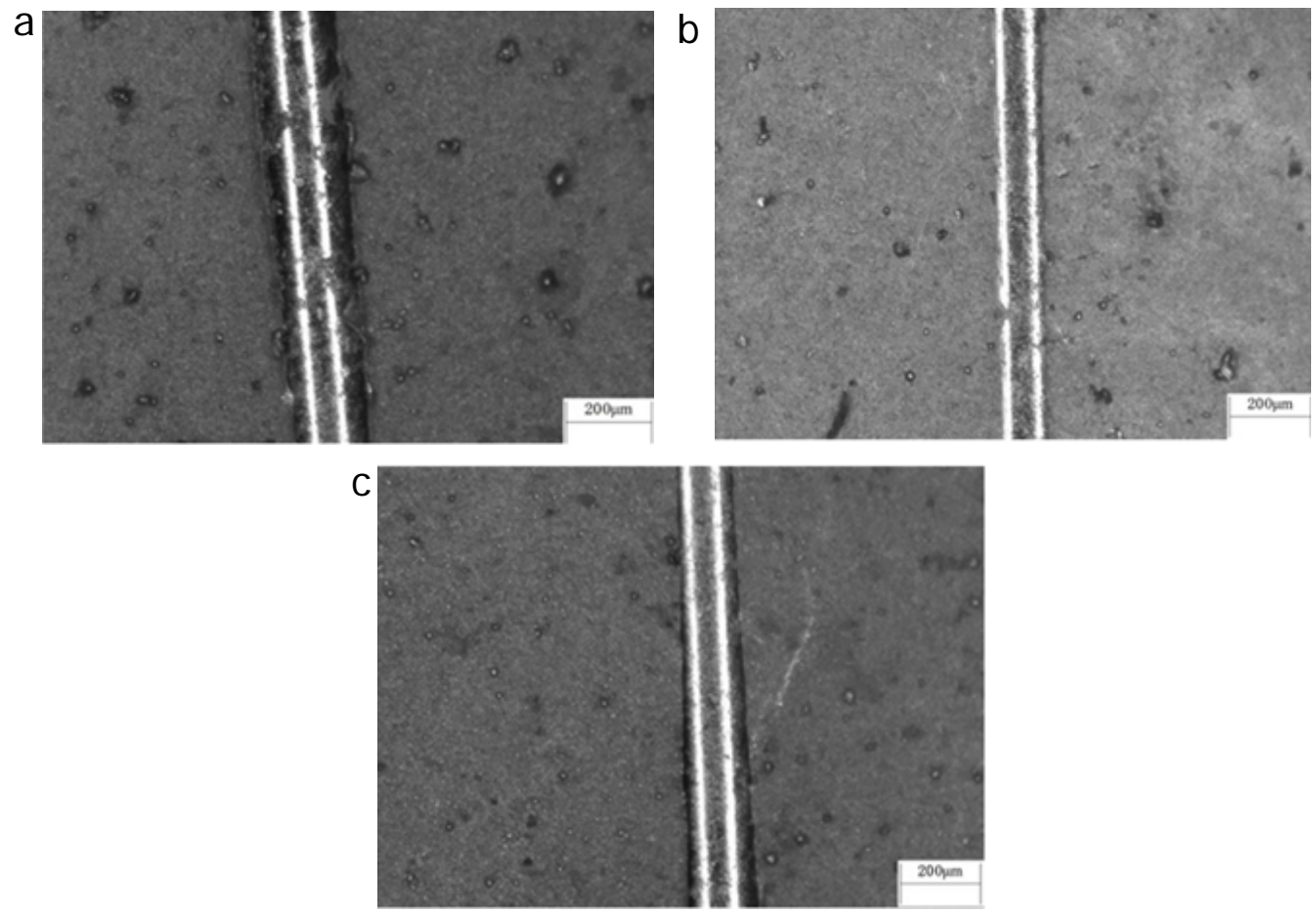

Fig. 6. The scratches photographs of coating obtained under different acting mold of current $(\times 50)$. (a) direct current (critical load is $25 \mathrm{~N}$ ); (b) positive pulse current (critical load is $40 \mathrm{~N}$ ); (c) reverse pulse current (critical load is $50 \mathrm{~N}$ ).

The influence of acting mold of current on micro hardness of coatings. The surface micro hardness of coating sobtained under different acting moldof current are shown in Fig. 7. It can be seen from the Fig. 7 that the coating obtained under reverse current electrode positing possess highest 
micro hardness and its average micro hardness reach $494.18 \mathrm{HV}_{0.1}$, the average micro hardness of coating obtained under positive current electrode positing reach $371.74 \mathrm{HV}_{0.1}$, the average micro hardness of coating obtained underdirect current electrode positing reach $322.68 \mathrm{HV}_{0.1}$.

The reasons for above results are as follows: during traditional direct current depositing process, due to the consumption of metal ions on surface of cathode could not be promptly supplemented and resulted in low concentration of discharge ions on the surface of cathode, further resulted in slow formation of crystal nucleus on the surface of electrode and fast growth of grain, coarse grains of coating; during positive pulse electrodepositing process, cathode polarization effect was enhanced in pulse on-time and ions on surface of cathode were supplemented from bath in pulse off-time, so that the concentration of metal ions in bath tends to be consistent, the concentration polarization effect was reduced, so the grain growth rate in coating was relatively slow, which was favorable to the grain refinement of deposited layer; during reverse pulse electrodepositing process, based on the characteristics of positive pulse electrodepositing, the concentration of metal ion on surface of cathode quickly recovered under action of negative pulse current, and the hydrogen inclusion in coating was oxidized, thereby the hydrogen embrittlement and internal stress in coating were reduced, so it is possible to obtain the coating having a finer grain size and a lower porosity.

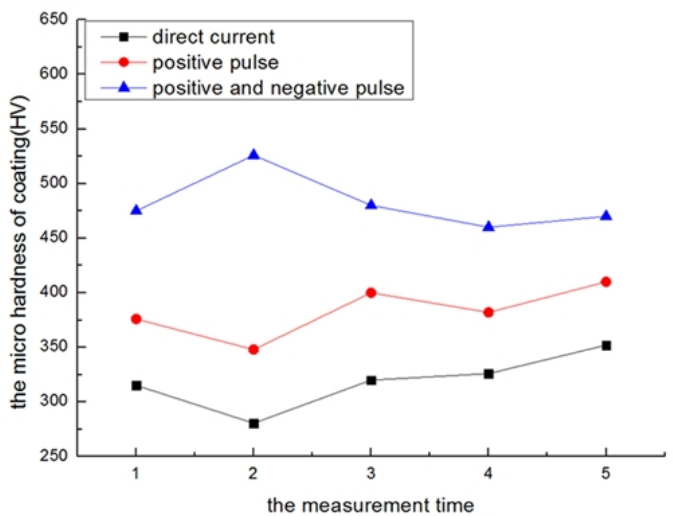

Fig.7. The surface micro hardness of coatings obtained under different acting mold of current.

The influence of acting mold of current on friction and wear performance of coating. The curves of friction coefficient-test time of coating obtained under different acting mold of current are shown in Fig. 8, the surface wear morphology of coatings obtained under different acting mold of current are shown in Fig. 9. It can be seen from the Fig. 8 that the friction coefficient of coating obtained under reverse current electrode positing was lower than the friction coefficient of coating obtained under positive pulse electrode positing, at the same time it was far lower than the friction coefficient of coating obtained under direct current electrode positing.

The reasons for above results are as follows: the effects of positive pulse electrodepositing made high degree of grain refinement, dense crystallization of coating and relatively large amount of co-depositing nano TiN particles, it would improve the hardness and enhance the ability of resistance to plastic deformation of Ni-nano TiN composite coating, so the friction coefficient of coating is relatively low; relative to the effects of positive pulse electrode positing, the effects of reverse current electrode positing made higher degree of grain refinement, more dense crystallization of coating and larger amount of co-depositing nano TiN particles, it would further improve the hardness of Ni-nanoTiN composite coating, so the friction coefficient of coating is lowest. 


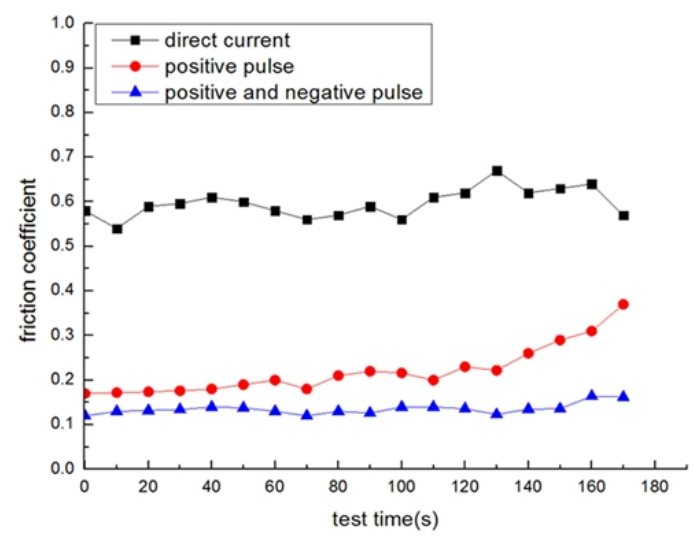

Fig. 8. The curves of friction coefficient-test time of coating obtained under different acting mold of current.

It can be seen from the Fig. 9 that there was serious abrasion on surface of coating obtained under direct current electrodepositing: large pieces of grinding, step-like wear marks, wide and deep furrow on wear surface accompanied pits and phenomena of massive spalling; there was greatly improved abrasion on surface of coating obtained under positive current electrode positing: significantly reduced pits and less phenomena of peeling, more sparse grinding marks; there was better wear resistance on surface of coating obtained under reverse current electrode positing: no large grinding appeared, wear scar became narrow and shallow.

a

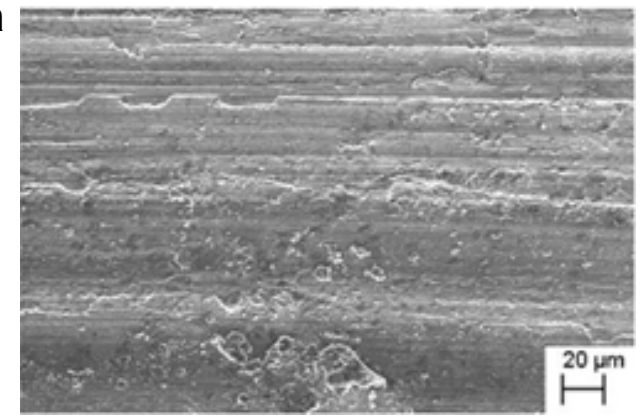

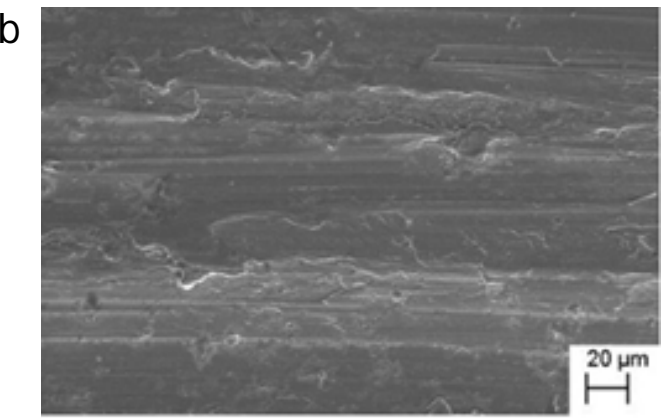

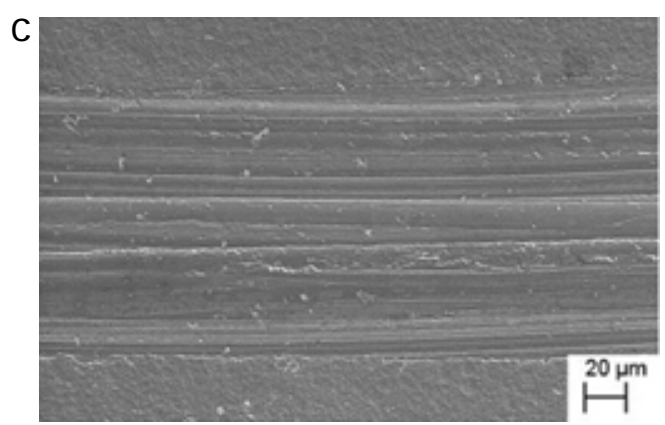

Fig. 9. The surface wear morphology (SEM) of coatings obtained under different acting mold of current. (a)direct current; (b) positive pulse current; (c) reverse pulse current.

The reasons for above results are as follows: firstly, the different refine degree and densification degree of matrix Ni crystal grain in composite coating led to difference of hardness and toughness of coating, thus it may influence the wear resistance performance of coating; secondly, the content of nano TiN particles in composite coating gradually increased and the defects of dislocation and twin crystal in composite coating also gradually increased with the change of acting mold of current (direct current, positive pulse current, reverse pulse current), these defects were conducive to improve the carrying capacity and anti-plastic deformation of coating; thirdly, co-depositing TiN particles may approximately form a transfer film within the matrix metal and its grain boundaries under condition 
of friction and wear, so it can effectively block movement of internal dislocation and sliding of grain boundary in composite coating; fourthly, nano TiN particles which evenly dispersed in coating could play the role of restructuring when coating surface was damaged, so that the coating could be strengthened, at the same time it also played a role of protection and lubrication for coating, thereby enhanced the wear resistance of coating[15-16].

\section{Conclusions}

(1)Compared with ordinary direct current depositing, pulse electrodepositing can obtain the Ni-nano TiN composite coating which possess more refined crystallization, more smooth surface, less cracks, higher binding force and higher micro hardness.

(2)Under the conditions of friction and wear, pits and peeling phenomena on surface of the coating obtained under positive current electrodepositing have a certain degree of reduction relative the coating obtained under direct current electrode positing, pits and peeling phenomena on surface of the coating obtained under reverse current electrodepositing have obviously reduction and the abrasion of the coating has been greatly improved.

(3)The reverse current electrodepositing can significantly improve the degree of grain refinement and density of Ni crystal, make the TiN nano particles more deposited in the coating and enhanced the effect of dispersion strengthening, so the performances of Ni-nano TiN composite coating may be significantly improved.

\section{Acknowledgements}

This work was supported by the Dalian Science and Technology Project (2015A11GX003) and the Science \&Technology Research Project of Education Department of Liaoning province (L2015029).

\section{References}

[1] X.Q. Ouyang, L.Y. Zhou, B. Yu, et al., Research status of electroplated Nickel-based alloy coating, Plating and Finishing. 36 (2014) 20-24. (in Chinese)

[2] Q.J. Xiang, Y. Liu, B.W. Liu, et al., Preparation of TiN-SiC Composite Ceramics, Cemented Carbide. 22(2005)78-81. (in Chinese)

[3] V. Zarghami, M. Ghorbani, Alteration of corrosion and nano mechanical properties of pulse electrodeposited $\mathrm{Ni} / \mathrm{SiC}$ nanocomposite coatings, Journal of Alloys and Compounds. 598(2014)236-242.

[4] X.B. Zhu, C. Chao, G.Q. Zheng, Electrodeposition and corrosion behavior of nanostructured Ni-TiN composite films, Transactions of Nonferrous Metals Society of China. 21 (2011)2216-2224. (in Chinese)

[5] L.T. Li, M.H. Wu, Y.G. Wang, Influences of NiCl2 content on electrodeposited Ni-Nano TiN composite coating process, Surface Technology. 44 (2015)7-12. (in Chinese)

[6] X.B. Zhu, C. Cai, G.Q. Zheng, Electrodeposition and characterization of nano-structured Ni-SiC composite films, Surface and Coatings Technology. 205 (2011)3448-3454.

[7] W. Wang, B. Yuan, C. Zhou, Formation and oxidation resistance of germanium modified silicide coating on Nb based in situ composites, Corrosion Science. 80 (2014)164-168. (in Chinese)

[8] M. Sabri, A.A. Sarabi, S.N. Kondelo, Materials Chemistry \& Physics. 136(2012)566-569. 
[9] F.F. Xia, Z.Y. Jia, M.H. Wu, The effect of sodium dodecyl sulfate surfactant on the electrodeposition of Ni-alumina composite coatings, Materials Science and Technology. 15(2008)779-781. (in Chinese)

[10]J.C. Witman, B.L. Bates, Y. Zhang, Electrolytic Co-deposition of Ni-CrAlY Composite Coatings Using Different Deposition Configurations, Materials and Manufacturing Processes. 31 (2016)1232-1237.

[11]H. Adelkhani, M.R. Arshadi, Properties of $\mathrm{Fe}-\mathrm{Ni}-\mathrm{Cr}$ alloy coatings by using direct and pulse current electrodeposition, Journal of Alloys and Compounds. 476(2009)234-237.

[12]M.H. Wu, J.H. Xue, H. Lv, Effects of heat treatment on wear resistance of nano Ni-TiN composite layer, Functional Materials. 41(2010)607-609. (in Chinese)

[13]F.F. Xia, M.H. Wu, Z.Y. Jia, Effect of nano TiN particles on Ni-TiN composite coatings, Heat Treatment of Metals. 32 (2007)62-64. (in Chinese)

[14] Y.J. Lee, J.Y. Park, Fabrication and characterization of multilayered nanoporus platinum films deposited by electroplating and nonionic surfactant molds, Applied Surface Science. 277 (2013)100-104.

[15] W. Sun, P. Zhang, K. Zhao, et al., Effect of graphite concentration on the friction and wear of $\mathrm{Ni}-\mathrm{Al} 2 \mathrm{O} 3$ /graphite composite coatings by a combination of electrophoresis and electrodeposition, Wear. 342(2015)172-180.

[16]N.P. Wasekar, S.M. Latha, M. Ramakrishna, et al., Pulsed electrodeposition and mechanical properties of Ni-W/SiC nano-composite coatings, Materials \& Design. 112 (2016)140-150. 